\title{
Real-world experience of CPX-351 as first-line treatment for patients with acute myeloid leukemia
}

\author{
Christina Rautenberg ${ }^{1,26}$, Friedrich Stölzel (D) ${ }^{2,26}$, Christoph Röllig ${ }^{2}$, Matthias Stelljes ${ }^{3}$, Verena Gaidzik ${ }^{4}$, Michael Lauseker (iD ${ }^{5}$, \\ Oliver Kriege ${ }^{6}$, Mareike Verbeek ${ }^{7}$, Julia Marie Unglaub ${ }^{8}$, Felicitas Thol ${ }^{9}$, Stefan W. Krause $\mathbb{D}^{10}{ }^{10}$, Mathias Hänel ${ }^{11}$, Charlotte Neuerburg ${ }^{12}$, \\ Vladan Vucinic ${ }^{13}$, Christian-Friedrich Jehn ${ }^{14}$, Julia Severmann ${ }^{15}$, Maxi Wass ${ }^{16}$, Lars Fransecky ${ }^{17}$, Jens Chemnitz $^{18}$, Udo Holtick ${ }^{19}$, \\ Kerstin Schäfer-Eckart ${ }^{20}$, Josephine Schröder ${ }^{21}$, Sabrina Kraus ${ }^{22}$, William Krüger ${ }^{23}$, Ulrich Kaiser ${ }^{24}$, Sebastian Scholl ${ }^{25}$, Kathrin Koch ${ }^{7}$, \\ Lea Henning ${ }^{1}$, Guido Kobbe ${ }^{1}$, Rainer Haas ${ }^{1}$, Nael Alakel ${ }^{2}$, Maximilian-Alexander Röhnert ${ }^{2}$, Katja Sockel (iD) ${ }^{2}$, Maher Hanoun ${ }^{15}$, \\ Uwe Platzbecker $^{13}$, Tobias A. W. Holderried ${ }^{12}$, Anke Morgner ${ }^{11}$, Michael Heuser (iD ${ }^{9}$, Tim Sauer ${ }^{8}$, Katharina S. Götze ${ }^{7}$, \\ Eva Wagner-Drouet ${ }^{6}$, Konstanze Döhner ${ }^{4}$, Hartmut Döhner ${ }^{4}$, Christoph Schliemann $^{3}$, Johannes Schetelig ${ }^{2}$, Martin Bornhäuser ${ }^{2}$, \\ Ulrich Germing ${ }^{1}$, Thomas Schroeder (iD) ${ }^{1,15,26 \bowtie}$ and Jan Moritz Middeke (iD ${ }^{2,26 \bowtie}$
}

(c) The Author(s) 2021

To investigate the efficacy and toxicities of CPX-351 outside a clinical trial, we analyzed 188 patients (median age 65 years, range 26-80) treated for therapy-related acute myeloid leukemia (t-AML, 29\%) or AML with myelodysplasia-related changes (AML-MRC, $70 \%$ ). Eighty-six percent received one, $14 \%$ two induction cycles, and $10 \%$ received consolidation (representing $22 \%$ of patients with $\mathrm{CR} / \mathrm{CRi}$ ) with $\mathrm{CPX}-351$. Following induction, CR/CRi rate was $47 \%$ including $64 \%$ of patients with available information achieving measurable residual disease (MRD) negativity $\left(<10^{-3}\right)$ as measured by flow cytometry. After a median follow-up of 9.3 months, median overall survival (OS) was 21 months and 1-year OS rate $64 \%$. In multivariate analysis, complex karyotype predicted lower response $(p=0.0001)$, while pretreatment with hypomethylating agents $(p=0.02)$ and adverse European LeukemiaNet 2017 genetic risk $(p<0.0001)$ were associated with lower OS. Allogeneic hematopoietic cell transplantation (allo-HCT) was performed in 116 patients (62\%) resulting in promising outcome (median survival not reached, 1-year OS 73\%), especially in MRD-negative patients $(p=0.048)$. With $69 \%$ of patients developing grade III/IV non-hematologic toxicity following induction and a day 30 -mortality of $8 \%$ the safety profile was consistent with previous findings. These real-world data confirm CPX-351 as efficient treatment for these high-risk AML patients facilitating allo-HCT in many patients with promising outcome after transplantation.

Blood Cancer Journal (2021)11:164; https://doi.org/10.1038/s41408-021-00558-5

\section{INTRODUCTION}

Acute myeloid leukemia (AML) can arise de novo, as therapyrelated complication following chemotherapy and/or ionizing radiation (t-AML) or from antecedent hematologic disorders [1]. The latter are also summarized as secondary AML (sAML) and account for approximately one quarter of all AML cases. AML with myelodysplasia-related changes (AML-MRC) is defined according to the WHO 2016 classification by the history of a myelodysplastic syndrome (MDS), signs of dysplasia, and/or MDS-related cytogenetic abnormalities. AML-MRC and SAML occur more frequently with advanced age and are associated with biologic properties such as adverse genetics and multidrug resistance phenotype,

\footnotetext{
${ }^{1}$ Department of Hematology, Oncology and Clinical Immunology, University Hospital Duesseldorf, Medical Faculty, Heinrich Heine-University, Duesseldorf, Germany. ${ }^{2}$ Medizinische Klinik und Poliklinik I, University Hospital Carl Gustav Carus Dresden, Technical University Dresden, Dresden, Germany. ${ }^{3}$ Department of Medicine A, University Hospital Münster, Münster, Germany. ${ }^{4}$ Department of Internal Medicine III, University Hospital of Ulm, Ulm, Germany. ${ }^{5}$ Institute for Medical Information Processing, Biometry and Epidemiology, Ludwig-Maximilians-University, Munich, Germany. ${ }^{6}$ Department of Medicine III, University Medical Center, Johannes Gutenberg University Mainz, Mainz, Germany. ${ }^{7}$ Department of Medicine III, Technical University of Munich, Munich, Germany. ${ }^{8}$ Department of Internal Medicine V, University Hospital Heidelberg, Heidelberg, Germany. ${ }^{9}$ Department of Hematology, Hemostasis, Oncology and Stem Cell Transplantation, Hannover Medical School, Hannover, Germany. ${ }^{10}$ Department V for Internal Medicine, University Hospital Erlangen, Erlangen, Germany. ${ }^{11}$ Department of Internal Medicine III, Klinikum Chemnitz, Chemnitz, Germany. ${ }^{12}$ Department of Oncology, Hematology and Rheumatology, University Hospital Bonn, Bonn, Germany. ${ }^{13}$ Leipzig: Department of Hematology and Cell Therapy, Medical Oncology, Hemostaseology, Leipzig, Germany. ${ }^{14}$ Department of Hematology, Oncology and Stem Cell Transplantation, Asklepios Clinic St. Georg, Hamburg, Germany. ${ }^{15}$ Department of Hematology and Stem Cell Transplantation, West German Cancer Center Essen, University Hospital Essen, Essen, Germany. ${ }^{16}$ Clinic and Policlinic for Internal Medicine IV, University Hospital Halle (Saale), Halle (Saale), Germany. ${ }^{17}$ Departmenf for Internal Medicine II, University Schleswig-Holstein, Kiel, Germany. ${ }^{18}$ Gemeinschaftsklinikum Mittelrhein GGmbH, Koblenz, Germany. ${ }^{19}$ Department I of Internal Medicine, University Hospital of Cologne, Cologne, Germany. ${ }^{20}$ Department of Internal Medicine V, Oncology and Hematology, Klinikum Nürnberg, Nürnberg, Germany. ${ }^{21}$ Clinic for Heaematology and Stem Cell Transplantation HELIOS Clinic Berlin-Buch GmbH, Berlin, Germany. ${ }^{22}$ Division of Hematology and Oncology, Department of Internal Medicine II, University of Würzburg, Medical Center, Würzburg, Germany. ${ }^{23}$ Clinic and Policlinic for Internal Medicine C, Hematology and Oncology, University of Greifswald, Greifswald, Germany. ${ }^{24}$ Department of Hematology and Oncology, St. Bernward Krankenhaus, Hildesheim, Germany. ${ }^{25}$ Department of Internal Medicine II, Hematology and Oncology, University Hospital Jena, Jena, Germany. ${ }^{26}$ These authors contributed equally: Christina Rautenberg, Friedrich Stölzel, Thomas Schroeder, Jan Moritz Middeke. ${ }^{凶}$ email: Thomas.schroeder@uk-essen.de; Janmoritz.middeke@ukdd.de
}

Received: 8 July 2021 Revised: 7 September 2021 Accepted: 17 September 2021

Published online: 04 October 2021 
which contribute to poor outcome after conventional therapy [2-5]. While attempts to improve outcome after induction therapy by addition of other agents or intensification of post-remission therapy have generally failed, higher remission rates and longer overall survival (OS) compared to conventional cytarabine plus daunorubicin chemotherapy $(7+3$ regimen) were recently observed following CPX-351 (Jazz Pharmaceuticals, Palo Alto, (A), a liposomal encapsulation of cytarabine and daunorubicin at a fixed 5:1 synergistic molar ratio $[6,7]$. The results from this phase-III trial, which investigated CPX-351 in 309 patients with AML-MRC or TAML aged 60-75 years, led to the approval of the drug combination by the health authorities in the USA 2017 and in Europe 2018 for adult patients with newly diagnosed AML-MRC or t-AML. Beside the aspect of age also several other issues, e.g. measurable residual disease (MRD), molecular subgroups, specific side effects as well as specific outcome parameters in the context of allogeneic hematopoietic cell transplantion (allo-HCT) were not addressed in the phase-III trial. Furthermore, in other settings data from real-world experiences with new therapies differed from clinical trial results $[8,9]$, suggesting that patients in clinical trials represent a selected cohort potentially limiting interpretation and translation of results to real-life patient care. Two recent retrospective analyses pointed towards some of these open questions $[10,11]$. Therefore, aiming to address these open aspects and to provide more clinical data and experience for CPX351 , we performed a real-world analysis of consecutive newly diagnosed patients with AML, who were treated in-label with CPX351 as first-line therapy.

\section{SUBJECTS AND METHODS \\ Study design}

For this retrospective analysis, we collected data from consecutive patients with newly diagnosed AML-MRC or t-AML, who were treated with CPX-351 according to the EMA label between 2018 and 2020 in 25 German centers participating in the Study Alliance Leukemia (SAL), German Cooperative Transplant Study Group, and the AML Study Group (AMLSG). Median number of patients included per center was 6 (range, 1-22 patients). Information about patient characteristics, treatment details including allo$\mathrm{HCT}$, and outcome were gathered using a specific, standardized data form sent to the participating centers. All patients in the participating centers treated with CPX-351 as first-line therapy for AML during this time period were reported. To ensure high data quality physicians' review of data and personal requests at respective centers was performed for all patients. The study was approved by the ethics committee of the Heinrich-HeineUniversity, Duesseldorf (approval number: 2020-877) and all patients gave written informed consent for scientific use of their data. Data lock for the analysis was 1 November 2020.

\section{Definitions and response criteria}

AML subtypes were categorized according to the criteria of the WHO 2016 classification [1]. Response, relapse, and genetic risk categories were defined according to the 2017 European LeukemiaNet (ELN) recommendations [12]. For the definition of complete remission without minimal residual disease $\left(\mathrm{CR}_{\mathrm{MRD}-}\right)$ we used information of $M R D$ results obtained by routine flow cytometry (FC)-based MRD monitoring performed at the participating site.

Conditioning intensity, the hematopoietic cell transplantation-specific comorbidity index (HCT-Cl), and graft-versus-host disease (GvHD) were defined as previously described [13-16]. Non-hematologic toxicity was graded by the treating physician using the National Cancer Institute Common Toxicity Criteria ( $\mathrm{NCl} \mathrm{CTC).}$

\section{Statistical analyses}

For categorical variables frequencies were displayed and differences were evaluated using cross-tabulation and Fisher's exact $t$-test, whereas for continuous variables medians (range) were summarized and the Mann-Whitney test was used to detect differences. OS for the entire cohort was calculated as the time from the first day of treatment with CPX351 to death from any cause or last follow-up in survivors. For the subgroup of patients undergoing allo-HCT OS was estimated as time between allo-HCT and death or date of last follow-up in surviving patients, while relapse-free survival (RFS) was calculated as time from allo-HCT until relapse or death without relapse censoring those patients, who had not relapsed until and were alive at date of last follow-up. Time-to-event curves were calculated by employing the Kaplan-Meier method and logrank tests were applied for univariate comparisons.

Furthermore, for the transplant cohort cumulative incidence of relapse (CIR) and non-relapse mortality (NRM) were considered as competing risks and calculated using cumulative incidence $(\mathrm{Cl})$ estimates employing Gray test for univariate comparisons. Those parameters, which influenced OS of the entire cohort in univariate analysis with $p<0.10$, were included into a multivariate analysis using a proportional hazard regression analysis (multiple Cox regression model). For factors associated with achievement of $C R / C R i$ a multinominal logistic regression analysis was applied.

In all analyses, a $p$ value $<0.05$ was considered to be significant. Statistical analyses were performed using GraphPad Prism ${ }^{\circledR} 7$ (GraphPad Software Inc., La Jolla, USA) and IBM SPSS Statistics (SPSS Inc. Chicago, IL) as well as R 3.5.1.

\section{RESULTS}

\section{Patients characteristics}

We analyzed data from 188 consecutive patients, who received CPX-351 induction chemotherapy as first-line therapy for AML between June 2018 and June 2020. Median age was 65 years (range $26-80$ years) and 46 patients (24\%) were $<60$ years. Most patients $(82 \%)$ had a good performance status as indicated by a Karnofsky index of $\geq 80 \%$, while the majority (82\%) exhibited comorbidities as indicated by intermediate or high HCT-Cl. A diagnosis of t-AML was present in $29 \%$ of patients, while $70 \%$ were diagnosed with AML-MRC. A total of 19 patients (10\%) had been treated with hypomethylating agents (HMA) for anteceding MDS before initiation of CPX-351. Karyotype abnormalities were present in $65 \%$ of patients including 44 patients (25\%) with a complex karyotype (CK). Considering the prognostically relevant mutations included in the 2017 ELN risk classification, ASXL1 $(n=$ $31,16 \%)$ was the most frequently mutated gene, followed by RUNX1 ( $n=24,13 \%), \operatorname{NPM1}(n=18,10 \%), \operatorname{TP} 53(n=14,7 \%)$, and FLT3-ITD ( $n=13,7 \%)$. Accordingly, 7, 33, and $60 \%$ of patients belonged to the favorable, intermediate, and adverse ELN 2017 genetic risk category, respectively. Detailed patients characteristics are summarized in Table 1.

\section{Treatment}

One-hundred and sixty-two patients (86\%) received one cycle of CPX-351 induction, while 26 patients (14\%) received two induction cycles (Table 2). Of these, 116 (62\%) underwent allo-HCT, which was performed without further therapy in 82 patients. In the remaining 34 patients at least one cycle of intermittent therapy consisting of either CPX-351 and/or AraC consolidation, HMA or salvage therapy was performed (Fig. 1). In 58 patients (31\%), who were alive beyond day +30 after induction, no allo-HCT was performed. Reasons for not proceeding to allo-HCT were mainly related to performance status, favorable disease risk, progressive disease, or patients' decision (Fig. 1). Of these non-transplanted patients, 25 patients received at least one other line of conventional therapy consisting of CPX-351 and/or AraC consolidation, HMA or salvage therapy, while in the remaining 33 patients no further therapy was performed (Fig. 1). Overall, at least one cycle of CPX-351 consolidation therapy was administered in 19 patients $(10 \%)$ of patients, which corresponds to $22 \%$ of patients with $\mathrm{CR} / \mathrm{CRi}$.

\section{Response after induction}

Following CPX-351- induction 47\% of evaluable patients achieved CR/CRi $(n=85)$ and $20 \%(n=35)$ morphologic leukemia-free state (MLFS), while $30 \%$ of evaluable patients $(n=53)$ did not respond. Among patients with $\mathrm{CR} / \mathrm{CRi}(n=85)$ after CPX-351 induction data 
Table 1. Patient characteristics $(n=188)$.

\begin{tabular}{|c|c|c|}
\hline Characteristics & $n$ & $\%$ \\
\hline Age, years (median, range) & $65(26-80)$ & \\
\hline \multicolumn{3}{|l|}{ Gender } \\
\hline Female & 70 & 37 \\
\hline Male & 118 & 63 \\
\hline \multicolumn{3}{|l|}{ Karnofsky $(n=165)$} \\
\hline$\geq 80$ & 135 & 82 \\
\hline$<80$ & 30 & 18 \\
\hline \multicolumn{3}{|l|}{$\mathrm{HCT}-\mathrm{Cl}(n=155)$} \\
\hline Low & 29 & 18 \\
\hline Intermediate & 55 & 35 \\
\hline High & 74 & 47 \\
\hline \multicolumn{3}{|l|}{ AML subtype $(n=186)$} \\
\hline AML-MRC & 131 & 70 \\
\hline $\mathrm{t}-\mathrm{AML}$ & 53 & 29 \\
\hline Other $^{\mathrm{a}}$ & 2 & 1 \\
\hline \multicolumn{3}{|l|}{2017 ELN genetic risk $(n=179)$} \\
\hline Favorable & 12 & 7 \\
\hline Intermediate & 59 & 33 \\
\hline Adverse & 108 & 60 \\
\hline \multicolumn{3}{|l|}{ Karyotype $(n=179)$} \\
\hline Normal & 63 & 35 \\
\hline Abnormal & 116 & 65 \\
\hline Complex & 44 & 25 \\
\hline Non-complex & 72 & 40 \\
\hline \multicolumn{3}{|l|}{ Molecular genetics } \\
\hline NPM1/n.d. & $18 / 11$ & $10 / 6$ \\
\hline FLT3-ITD/n.d. & $13 / 13$ & $7 / 7$ \\
\hline ASXL1/n.d. & $31 / 24$ & $16 / 13$ \\
\hline TP53/n.d. & $14 / 26$ & $7 / 14$ \\
\hline RUNX1/n.d. & $24 / 20$ & $13 / 11$ \\
\hline BM blast count at diagnosis (median, range) & $38(7-99)$ & \\
\hline PB blast count at diagnosis (median, range) & $10(0-92)$ & \\
\hline WBC at diagnosis, $\times 10^{3} / \mu \mathrm{l}$ (median, range) & $3.8(0.6-330)$ & \\
\hline No treatment prior $\mathrm{CPX}$ & 169 & 90 \\
\hline Pretreatment with $\mathrm{HMA}$ & 19 & 10 \\
\hline
\end{tabular}

$A M L$ acute myeloid leukemia, $A M L-M R C$ AML with myelodysplasia-related changes, BM bone marrow, ELN European Leukemia Net, HCT-Cl hematopoietic cell transplantation-specific comorbidity index, HMA hypomethylating agents, n.d. not done, $n$ number, $P B$ peripheral blood, $t-A M L$ therapyrelated $A M L, W B C$ white blood cells.

Numbers in parentheses display patients with available information.

a Secondary AML evolving from systemic mastocytosis $(n=1)$ and blastic plasmacytoid dendritic cell neoplasm (BPDCN) with antecedent history of chronic myelomonocytic leukemia (CMML) $(n=1)$.

on MRD measured by flow cytometry were available for 36 patients (42\%) demonstrating MRD negativity in $64 \%$ of the patients $(n=23)$ (Table 2). CR/CRi rate in patients with complex karyotype $(n=44)$ and in those with TP53 mutation $(n=14)$ were $33 \%$ and $54 \%$, respectively.

Given the limited data in patients treated with CPX-351 so far $[6,10,11]$, we aimed to identify predictors for achievement of CR/ $\mathrm{CRi}$. In univariate analyses, we observed higher $\mathrm{CR} / \mathrm{CRi}$ rates in female patients, in those without complex karyotype and in those patients without HMA pretreament (Supplementary Table 1). In
Table 2. Treatment characteristics, response, and toxicity.

\begin{tabular}{llc|}
\hline Characteristics & $n$ & $\%$ \\
\hline Follow-up, months (median, range) & $9.3(0.2-26.1)$ & \\
$\begin{array}{l}\text { No. of induction cycles with CPX } \\
\text { (median, range) }\end{array}$ & $1(1-2)$ \\
\hline ANC recovery ${ }^{\mathrm{a}}$ in patients with CR/CRi after CPX-351 induction $(n=83)$ \\
\hline Yes & 79 & 95 \\
\hline No & 4 & 5 \\
\hline Time, days (median, range) & $33(6-99)$ & \\
\hline
\end{tabular}

Platelet recovery ${ }^{\mathrm{b}}$ in patients with CR/CRi after CPX-351 induction ( $n=$ 83)

$\begin{array}{lll}\text { Yes } & 76 & 92 \\ \text { No } & 7 & 8\end{array}$

Time, days (median, range) 30 (7-77)

Response after induction $(n=179)$

CR/CRi $\quad 85 \quad 47$

MLFS $\quad 35 \quad 20$

Refractory disease $\quad 53 \quad 30$

Early death ( $<30$ days) without response $\quad 6 \quad 3$

Evaluation

MRD available in case of CR/CRi $\quad 36 \quad 42^{\mathrm{C}}$

MRD negative $\quad 23 \quad 64^{\mathrm{d}}$

Grade III/IV non-hematologic toxicities $(n=188)$

Infection $\quad 41 \quad 22$

GI (mucositis, nausea, vomiting) $\quad 7 \quad 4$

Bleeding $\quad 7 \quad 4$

Renal failure $\quad 5 \quad 3$

Febrile neutropenia $\quad 28 \quad 15$

$\begin{array}{lll}\text { Pneumonia } & 42 & 22\end{array}$

Mortality on d30 after CPX-351 induction $(n=176)^{\mathrm{e}}$

$\begin{array}{lll}\text { Alive } & 162 & 92 \\ \text { Dead } & 14 & 8\end{array}$

ANC absolute neutrophile count, $C R$ complete remission, $C R i$ complete remission with incomplete hematologic recovery, $d$ day, Gl gastrointestinal, MLFS morphologic leukemia-free state, MRD minimal residual disease, $n$ number, $S D$ stable disease, $P D$ progressive disease, n.d. not done.

Numbers in parentheses display patients with available information.

${ }^{a}$ Defined as ANC $>500 / \mu \mathrm{l}$.

${ }^{\mathrm{b}}$ Defined as platelet count $>50,000 / \mu \mathrm{l}$.

${ }^{\mathrm{C}} \mathrm{Of}$ the 85 patients with $\mathrm{CR} / \mathrm{CRi}$.

${ }^{\mathrm{d}}$ Of the 36 patients with available MRD results.

ePatients proceeding to allo-HSCT before $\mathrm{d} 30$ following CPX-351 induction have been excluded.

multivariate analysis, only a non-complex karyotype $(p=0.0001)$ predicted for a higher CR/CRi rate (Table 3 ).

\section{Safety}

In patients with $\mathrm{CR} / \mathrm{CRi}$ recovery to an absolute neutrophil count (ANC) $\geq 500 / \mu \mathrm{l}$ and platelet count $\geq 50.000 / \mu \mathrm{l}$ was observed in $95 \%$ and $92 \%$ of patients, respectively (Table 2 ). Median time to ANC and platelet recovery was 33 days (range: 6-99 days) and 30 days (range: 7-77 days), respectively.

Regarding non-hematologic toxicity, adverse events $(A E) \geq$ grade III were reported in 130 patients (69\%). As indicated in Table 2, these were mainly related to infectious complications, while gastrointestinal side effects and bleeding occurred rather infrequently. Of note, patients with leukocyte counts $>20 \mathrm{G} / \mathrm{L}$ at diagnosis had a significantly higher frequency of grade III/IV AE as compared to those with leukocytes $<20 \mathrm{G} / \mathrm{L}$ (74\% vs. $50 \%$, 


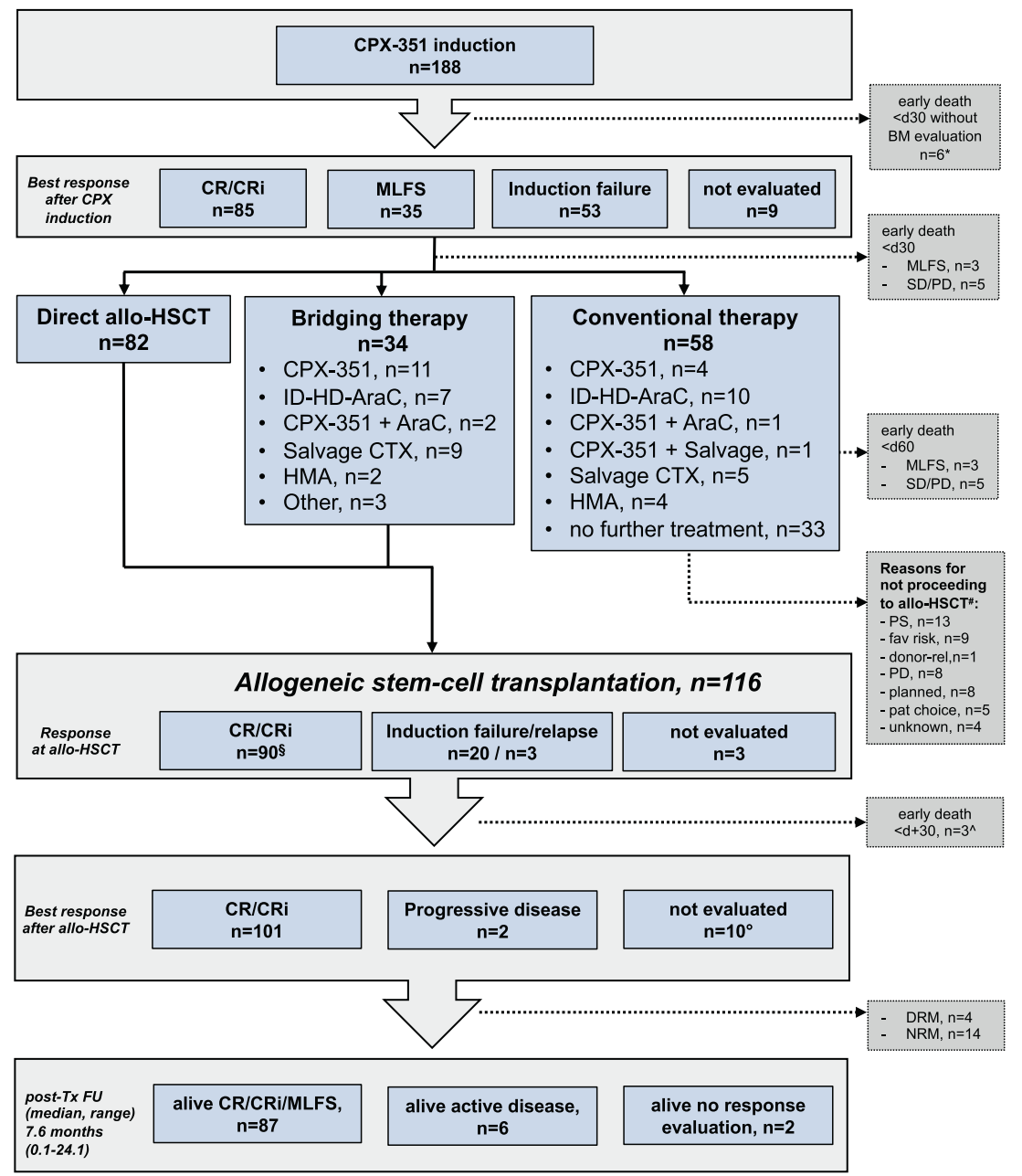

${ }^{*} \mathrm{n}=1$ received allo-HSCT 18 days after 1 st day of CPX-351 induction

$\#_{n}=2$ no information whether patient proceeded to allo-HSCT or not

$\S_{\mathrm{n}}=4$ after receiving salvage-CTX and $\mathrm{n}=1$ had no response evaluation after CPX-351 induction

$\wedge_{n}=2$ had active disease prior allo-HSCT

o $n=6$ had post-transplant follow-up $<30$ days and no $B M$ evaluation

Fig. 1 Flow chart depicting treatment, response, and outcome of the study population. Allo-HSCT allogeneic hematopoietic stem cell transplantation, BM bone marrow, CR complete remission, CRi complete remission with incomplete hematologic recovery, CTX chemotherapy, DRM disease-related mortality, d day, fav favorable, FU follow-up, HD-AraC high-dose cytarabine, ID-AraC intermediate dose cytarabin, MLFS morphologic leukemia-free state, NRM non-relapse mortality, pat patient, PD progressive disease, PS performance status, rel related, SD stable disease, Tx transplant.

$p=0.01)$. The 30-day early death rate was $8 \%$ in the entire cohort and was significantly higher in patients $\geq 65$ years $(11 \%$ vs. $3 \%, p$ $=0.047)$.

\section{Overall survival of the entire cohort}

With a median follow-up (FU) of 9.3 months (range: 0.2-26.1 months) median OS of the entire cohort was 21 months and estimated 1-year OS was $64 \%$ (95\% Cl 55-72\%, Fig. 2). In univariate analysis, pretreatment with HMA, the presence of an abnormal or complex karyotype, adverse ELN genetic risk, age $\geq 65$ years, MRD positivity after induction, and not undergoing allo-HCT were associated with inferior OS (Supplementary Table 2). In multivariate analysis, pretreatment with HMA $(p=0.02)$ and adverse ELN genetic risk $(p<0.0001)$ retained their negative impact on OS (Table 3).

\section{Allo-HCT}

One-hundred and sixteen (62\%) patients underwent allo-HCT after CPX-351 induction with a median time from start of induction to transplantion of 70 days (range: $11-215$ days). At the time of transplant 20\% $(n=23)$ of patients had active disease ( $>5 \%$ BM blasts, induction failure $n=20$, relapse $n=3)$, while $80 \%(n=90)$ were in first remission (Fig. 1). The latter included 26 of the 35 patients initially categorized as MLFS at first remission control, which were classified as CRi $(n=16)$ or CR $(n=10)$ at the time of transplant. Among patients with $\mathrm{CR} / \mathrm{CRi}$ at transplant flowcytometry-measured MRD status was available in 36 patients with 23 patients (64\%) exhibiting MRD negativity at the time of transplant. Detailed information on transplant characteristics are summarized in Table 4 and Supplementary Tables 3 and 4 . The majority of patients $(n=95,82 \%)$ received a graft from an unrelated donor following a reduced-intensity conditioning (RIC), which was used in all patients except for one.

A primary graft failure was observed in two patients (2\%). Median time to ANC and platelet recovery was 16 days (range: 7-70 days) and 15 days (range: 9-45 days), respectively. A sinusoidal obstruction syndrome (SOS) was reported in one patient $(1 \%)$. Acute GvHD (aGvHD) occurred in $50 \%$ of patients and aGVHD $\geq$ grade III was observed in $13 \%$ of patients, while chronic GvHD (cGvHD) was seen in $21 \%$ of patients including $4 \%$ 
Table 3. Outcome after induction with CPX-351, multivariate analysis.

\begin{tabular}{|c|c|c|c|c|c|c|}
\hline \multirow{2}{*}{ Variable } & \multicolumn{3}{|c|}{ Overall survival } & \multicolumn{3}{|c|}{ Response rate } \\
\hline & $P$ & & HR & $P$ & & HR \\
\hline \multicolumn{7}{|c|}{ Prior treatment with HMA } \\
\hline Yes & 0.02 & & $2.4[1.1-5.3]$ & & n.s. & \\
\hline \multicolumn{7}{|l|}{ No } \\
\hline \multicolumn{7}{|c|}{ ELN risk stratification } \\
\hline Adverse & $<0.0001$ & & $4.2[1.9-8.9]$ & & - & \\
\hline \multicolumn{7}{|c|}{ Favorable/intermediate } \\
\hline \multicolumn{7}{|c|}{ Karyotype } \\
\hline Complex & & - & & 0.0001 & & $4.3[1.9-9.2]$ \\
\hline \multicolumn{7}{|c|}{ Not complex } \\
\hline \multicolumn{7}{|l|}{ NPM1 } \\
\hline wt & & n.s. & - & & & \\
\hline \multicolumn{7}{|l|}{ mut } \\
\hline \multicolumn{7}{|l|}{ TP53 } \\
\hline mut & & n.s. & - & & & \\
\hline \multicolumn{7}{|l|}{ wt } \\
\hline \multicolumn{7}{|c|}{ Age at diagnosis (median) } \\
\hline$\geq 65$ & & n.s. & - & & & \\
\hline \multicolumn{7}{|l|}{$<65$} \\
\hline \multicolumn{7}{|l|}{ Gender } \\
\hline Female & & - & n.s. & & & \\
\hline Male & & & & & & \\
\hline
\end{tabular}

ELN European Leukemia Net, HMA hypomethylating agents, HR hazard ratio, mut mutated, n.s. not significant, $P p$ value, wt wild type.

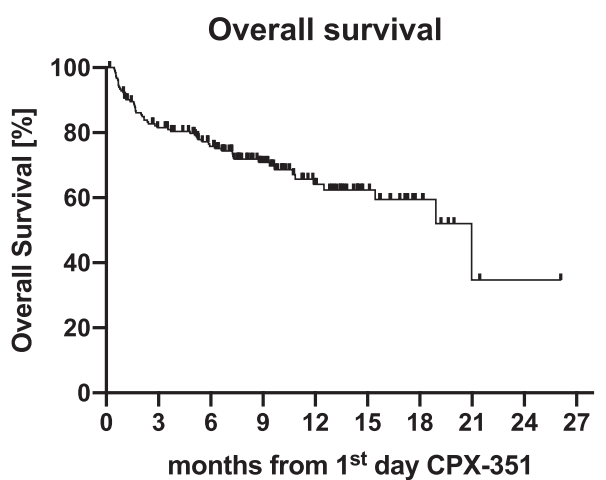

Fig. 2 Overall (OS) of the entire cohort. After a median follow-up of 9.3 months (range: 0.2-26.1 months) median OS of the entire cohort $(n=188)$ was 21 months and estimated 2 -year OS was $35 \%$. OS for the entire cohort was calculated as the time from the first day of treatment with CPX-351 to death from any cause or last follow-up in survivors.

with severe cGvHD. In evaluable patients, day 100 mortality was $7 \%$.

With a median FU of 7.6 months (range: $0.1-24.1$ months) 1-year OS, RFS, CIR, and NRM probabilities 73\%, 71\%, 23\%, and $12 \%$, respectively (Fig. 3). In a next step, similar to the entire cohort, we aimed to identify predictors for outcome after allo-HCT. In univariate analyses, only MRD positivity prior to transplant was associated with shorter OS (Fig. 4), while diagnosis of t-AML and active disease at time of transplant adversely impacted RFS (Supplementary Table 5). A complex karyotype, previous treatment with $\mathrm{HMA}$, and a mismatched donor were associated with a higher relapse incidence in univariate analyses (Supplementary
Table 5). Due to the low number of events no parameter associated with NRM could be ascertained. Together with the limited availability of information regarding some parameters in all patients this also impeded multivariate analyses.

\section{DISCUSSION}

Here, we report data from 188 patients treated in-label with CPX351 as first-line treatment for the diagnosis of AML. This patient number, which represents to the best of our knowledge the largest cohort outside a clinical trial reported so far, enabled us to provide sufficient data on response and outcome, course of treatment including allo-HCT and to identify predictors for response and survival.

First, our results show that in a real-life setting CPX-351 is used in patients exhibiting high-risk characteristics, which are in many aspects comparable to those in the phase-III trial, but also to those reported in two other real-world data sets of 103 and 71 patients $[6,10,11]$. This applies to the median age, performance status, comorbidities, frequency of AML subtypes, BM blast count, cytogenetics, molecular abnormalities, and NCCN/ELN risk categorization. Indeed, similar to the other cohorts $[6,10,11]$ secondary-type mutations [17] in genes like $A S X L 1, R U N X 1$, and TP53 mutations represented the most common alterations, but most interestingly neither influenced response or survival following CPX-351 treatment. This is in contrast to the pivotal and also the French study, in which at least TP53 mutations predicted for inferior response. Still, it has to be taken into account that molecular data could not be comprehensively analyzed in all patients. The higher frequency of patients with HMA pretreatment in the prospective trial compared to the three real-world series might be related to the approval of HMA also for low-risk MDS in the US. 
Table 4. Transplant characteristics, toxicity and outcome.

\begin{tabular}{|c|c|c|}
\hline Characteristics & $n$ & $\%$ \\
\hline $\begin{array}{l}\text { Time between 1st day of CPX-351 and allo-HCT, } \\
\text { days (median, range) }\end{array}$ & $70(11-215)$ & \\
\hline Median age at transplant & $64(24-79)$ & \\
\hline \multicolumn{3}{|l|}{ HLA-matching $(n=116)$} \\
\hline MUD & 74 & 64 \\
\hline MRD & 13 & 11 \\
\hline MMUD & 21 & 18 \\
\hline Haploidentical & 8 & 7 \\
\hline \multicolumn{3}{|l|}{ Donor gender $(n=112)$} \\
\hline Male & 78 & 70 \\
\hline Female & 34 & 30 \\
\hline \multicolumn{3}{|l|}{ CMV-status (donor/recipient) $(n=111)$} \\
\hline pos/pos & 44 & 40 \\
\hline pos/neg & 7 & 6 \\
\hline neg/neg & 35 & 32 \\
\hline neg/pos & 25 & 22 \\
\hline \multicolumn{3}{|l|}{ Conditioning intensity $(n=116)$} \\
\hline Reduced intensity & 115 & 99 \\
\hline Standard dose & 1 & 1 \\
\hline \multicolumn{3}{|l|}{ Stem cell source $(n=113)$} \\
\hline PBSC & 110 & 97 \\
\hline BM & 3 & 3 \\
\hline \multicolumn{3}{|l|}{ In vivo T cell depletion $(n=110)$} \\
\hline Yes & 69 & 63 \\
\hline No & 41 & 37 \\
\hline \multicolumn{3}{|l|}{ Engraftment $(n=114)$} \\
\hline No & 2 & 2 \\
\hline Yes & 112 & 98 \\
\hline \multicolumn{3}{|l|}{ ANC recovery after allo-HCT $(n=101)$} \\
\hline Yes & 100 & 99 \\
\hline No & 1 & 1 \\
\hline Time, days (median, range) & $16(7-70)$ & \\
\hline \multicolumn{3}{|l|}{ Platelet recovery after allo-HCT $(n=101)$} \\
\hline Yes & 96 & 96 \\
\hline No & 5 & 5 \\
\hline Time, days (median, range) & $15(9-45)$ & \\
\hline \multicolumn{3}{|l|}{ Acute GvHD $(n=113)$} \\
\hline Yes & 56 & 50 \\
\hline No & 57 & 50 \\
\hline$\geq$ Grade III & 15 & 13 \\
\hline \multicolumn{3}{|l|}{ Chronic GvHD $(n=110)$} \\
\hline Yes & 23 & 21 \\
\hline No & 87 & 79 \\
\hline Severe & 4 & 4 \\
\hline \multicolumn{3}{|l|}{ Grade III/IV non-hematologic toxicities $(n=116)$} \\
\hline Infection & 36 & 31 \\
\hline GI (mucositis, nausea, vomiting) & 11 & 9 \\
\hline Bleeding & 2 & 2 \\
\hline Renal failure & 8 & 7 \\
\hline Febrile neutropenia & 2 & 2 \\
\hline SOS & 1 & 1 \\
\hline Relapse after allo-HCT & & \\
\hline
\end{tabular}

\begin{tabular}{|lll|}
\hline Table 4 continued & & \\
\hline $\begin{array}{l}\text { Characteristics } \\
\quad \text { Yes }\end{array}$ & $n$ & $\%$ \\
\hline $\begin{array}{l}\text { Time between allo-HCT and relapse, days } \\
\text { (median, range) }\end{array}$ & 11 & 11 \\
\hline $\begin{array}{l}\text { No } \\
\text { Posttransplant day }+100 \text { mortality }(n=116)\end{array}$ & 90 & 89 \\
\hline $\begin{array}{l}\text { Dead } \\
\text { Alive }\end{array}$ & 8 & 7 \\
\hline $\begin{array}{l}\text { Alive, evaluated <day }+100 \\
\text { Posttransplant follow-up, months } \\
\text { (median, range) }\end{array}$ & 95 & 82 \\
\hline
\end{tabular}

ANC absolute neutrophile count, Allo-HCT allogeneic hematopoietic cell transplantation, $B M$ bone marrow, $C M V$ cytomegalovirus, $G$ l gastrointestinal, GVHD graft-versus-host disease, HLA human leukocyte antigen, MMUD mismatched unrelated donor, MRD matched related donor, MUD matched unrelated donor, neg negative, PBSC peripheral blood stem cells, pos positive, SOS sinusoidal obstruction syndrome.

Numbers in parentheses display patients with available information.

Despite many similarities in patients characteristics the treatment course substantially differed between our cohort, the population in the prospective trial, and the two real-world series $[6,10,11]$. Potentially due to the trial design a higher proportion of patients in the prospective study (31\% compared to $5-14 \%$ ) received a second induction with CPX-351. In contrast, in our cohort a lower frequency of patients received at least one CPX-351 consolidation (10\%) compared to the three other populations (ranging from 32 to $46 \%$ ). This is probably a consequence of a much higher rate of patients undergoing allo-HCT in our cohort $(62 \%$ vs. $27-35 \%)$, with the majority proceeding directly to transplant after induction. The latter might be related to a higher frequency of rapidly available donors and/or a general treatment philosophy to proceed to transplant in as many patients as possible. Additionally, since a similar frequency of 20 patients in our cohort received conventional intermediate/high-dose cytarabine for consolidation, the low rate of CPX-351 consolidation might also be related in parts to a greater familiarness of physicians with conventional cytarabine consolidation.

Following induction, we observed an identical CR/CRi rate of $47 \%$ like in the prospective trial, while CR/CRi rates were 70 and $59 \%$ in the Italian and French patients [6, 10, 11]. Response assessment was not performed at a pre-defined time point in the retrospective analyses. Of note, from 35 patients initially classified as MLFS, 26 turned into CR/CRi at a later point. Thus, from these data a CR/CRi rate of $47 \%$ can realistically be expected after CPX351 induction. If even a higher $C R / C R i$ might be reached, will be prospectively addressed in the ongoing AMLSG 30-18 trial (NCT03897127).

The survival benefit of CPX-351 in the phase-III trial in all patients and those achieving CR/CRi but not undergoing allo-HCT as well as the likelihood of proceeding to allo-HCT suggest the potential for achievement of deeper responses with CPX-351 $[6,18]$. While the impact of MRD was not prospectively addressed in this study, we observed MRD negativity detected by MFC in $64 \%$ of CR/CRi patients after induction. This appears not only similar to a rate of MRD negativity at the $10^{-3}$ sensitivity threshold (assessed with MFC or molecular methods) in $57 \%$ of patients of the French series [10], but also seems to be higher than the rate of MRD negativity observed after conventional $3+7$ induction in such a high-risk population [19]. Accounting for the retrospective character and the limited patient number with available MRD information, the impact of MRD requires prospective and standardized investigation as envisaged, for example, in the AMLSG 30-18 trial. 
Overall survival

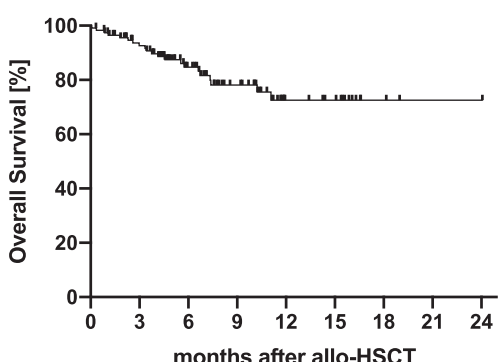

Relapse-free survival

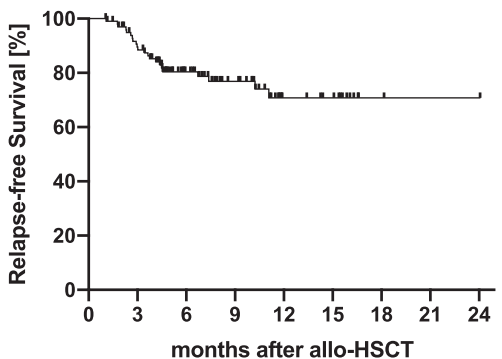

Cumulative incidence of relapse $I$ Non-relapse mortality

Fig. 3 Overall (OS), relapse-free survival (RFS), cumulative incidences of relapse (CIR), and non-relapse mortality (NRM) of the transplant cohort. With a median FU of 7.6 months (range: 0.1-24.1 months) estimated 2-year OS, RFS, CIR, and NRM probabilities of the entire cohort were $73 \%, 71 \%, 23 \%$, and $12 \%$, respectively. OS was estimated as time between allo-HSCT and death or date of last follow-up in surviving patients, while RFS was calculated as time from allo-HSCT until relapse or death without relapse censoring those patients, who had not relapsed until and were alive at date of last follow-up. CIR and NRM were considered as competing risks and calculated using cumulative incidence $(\mathrm{Cl})$ estimates employing Gray test for univariate comparisons.

Overall survival

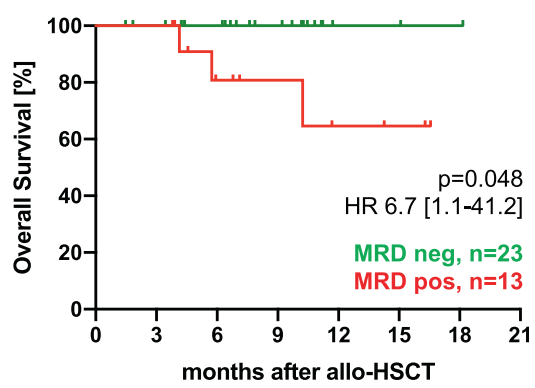

Relapse-free survival

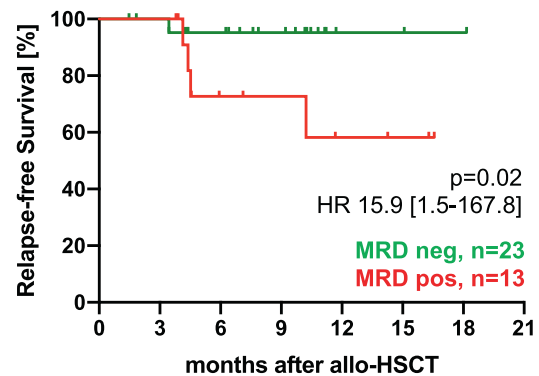

Fig. 4 Posttransplant outcome of patients depending on pre-transplant MRD status assessed by flow cytometry (FC). Among patients with CR/CRi $(n=85)$ after CPX-351-based induction data on MRD estimated by FC were available for 36 patients (42\%) representing MRD negativity in $64 \%$ of the patients $(n=23)$. The figure represents outcome in terms of overall and relapse-free survival for patients with pretransplant $\mathrm{CR}_{\mathrm{MRD}-}\left(n=23\right.$, green line) and $\mathrm{CR}_{\mathrm{MRD}+}(n=13$, orange line).

The median survival of 21 and 16.1 months in our and the French cohort and the 1-year OS rate of $73 \%$ support the impression from the prospective trial that CPX-351 confers a survival benefit in such a high-risk population $[6,18]$. Still, we have to acknowledge the short median follow-up of 9.3 months of our patients, which is comparable with the French ( 8.6 months) and Italian (11 months) cohorts, but definitively represents a limitation of our analysis.

Besides its efficacy in terms of remission induction, a favorable toxicity profile and a high rate of allo-HCT with favorable outcome after transplant contributed to the survival benefit in the phase-III trial. The low frequency of grade III/IV adverse events, especially gastrointestinal, expectable times to neutrophil and platelet recovery without excess of severe infectious complications and a day 30 mortality of $8 \%$ observed in our analysis confirm the favorable toxicity profile of CPX-351. This probably also contributed to the high rate of patients proceeding to allo-HCT with a RIC-based transplant. Indeed, we observed engraftment in all but two patients, hematopoietic recovery in the vast majority within an appropriate time, only one SOS and a low day 100 mortality of $7 \%$. Together with a low relapse rate, especially in those patients transplanted in MRD-negative $C R$, this translated into a promising survival after transplant as already previously reported $[6,10,11]$. Finally, the opportunity of a growing number of treatment alternatives for patients with high-risk AML such as venetoclax [20] or glasdegib [21] renders the choice of optimal treatment more complex. Aiming to support physicians in this process, we identified previous HMA treatment and 2017 ELN adverse-risk categorization as negative predictors for survival and a complex karyotype for response in multivariate analysis. While in the prospective trial patients without HMA pretreatment also had also a survival benefit, this could not be supported by the results from the French and Italian consortia [6, 10, 11]. In the French cohort TP53 and PTPN11 mutations were associated with lower response rates, while splicosome mutations correlated with higher OS. In the Italian cohort no predictor for response could be ascertained, and performance of allo-HCT was the only factor associated with better survival. Therefore, the predictive and prognostic impact of patient- and disease-related factors including molecular characteristics in the context of CPX-351 treatment remains still elusive [22] and requires prospective investigation optimally within a randomized trial.

In summary, our data show that under real-world circumstances CPX-351 is an efficient and clinically relevant treatment option for patients with AML-MRC and t-AML. Given the promising outcome following transplant, a combined approach applying an effective induction with CPX-351 and inducing an MRD-negative CR followed by the subsequent allo-HCT without further delay [23] may constitute the treatment approach with the highest probability of cure for this high-risk population.

\section{REFERENCES}

1. Arber DA, Orazi A, Hasserjian R, Thiele J, Borowitz MJ, Le Beau MM, et al. The 2016 revision to the World Health Organization classification of myeloid neoplasms and acute leukemia. Blood. 2016;127:2391-405.

2. Nagel G, Weber D, Fromm E, Erhardt S, Lübbert M, Fiedler W, et al. GermanAustrian AML Study Group (AMLSG) Epidemiological, genetic, and clinical 
characterization by age of newly diagnosed acute myeloid leukemia based on an academic population-based registry study (AMLSG BiO). Ann Hematol. 2017;96:1993-2003.

3. Hulegårdh E, Nilsson C, Lazarevic V, Garelius H, Antunovic P, Rangert Derolf $\AA$, et al. Characterization and prognostic features of secondary acute myeloid leukemia in a population-based setting: a report from the Swedish Acute Leukemia Registry. Am J Hematol. 2015;90:208-14.

4. Kayser S, Döhner K, Krauter J, Köhne $\mathrm{CH}$, Horst HA, Held G, et al. The impact of therapy-related acute myeloid leukemia (AML) on outcome in 2853 adult patients with newly diagnosed AML. Blood. 2011;117:2137-45.

5. Granfeldt Østgård LS, Medeiros BC, Sengeløv H, Nørgaard M, Andersen MK, Dufva $\mathrm{IH}$, et al. Epidemiology and clinical significance of secondary and therapy-related acute myeloid leukemia: a national population-based cohort study. J Clin Oncol. 2015;33:3641-9.

6. Lancet JE, Uy GL, Cortes JE, Newell LF, Lin TL, Ritchie EK. et al. CPX-351 (cytarabine and daunorubicin) liposome for injection versus conventional cytarabine plus daunorubicin in older patients with newly diagnosed secondary acute myeloid leukemia. J Clin Oncol. 2018;36:2684-92.

7. Mayer LD, Tardi P, Louie AC. CPX-351: a nanoscale liposomal co-formulation of daunorubicin and cytarabine with unique biodistribution and tumor cell uptake properties. Int J Nanomed. 2019;14:3819-30.

8. Winters AC, Gutman JA, Purev E, Nakic M, Tobin J, Chase S, et al. Real-world experience of venetoclax with azacitidine for untreated patients with acute myeloid leukemia. Blood Adv. 2019;3:2911-9.

9. Juliusson G, Lazarevic V, Hörstedt AS, Hagberg O, Höglund M, Swedish Acute Leukemia Registry G. Acute myeloid leukemia in the real world: why populationbased registries are needed. Blood. 2012;119:3890-9.

10. Chiche E, Rahmé R, Bertoli S, Dumas PY, Micol JB, Hicheri $Y$, et al. Real-life experience with CPX-351 and impact on the outcome of high-risk AML patients: a multicentric French cohort. Blood Adv. 2021;5:176-84.

11. Guolo F, Fianchi L, Minetto P, Clavio M, Gottardi M, Galimberti S. et al. CPX-351 treatment in secondary acute myeloblastic leukemia is effective and improves the feasibility of allogeneic stem cell transplantation: results of the Italian compassionate use program. Blood Cancer J. 2020;10:96.

12. Döhner H, Estey $E$, Grimwade $D$, Amadori $S$, Appelbaum FR, Büchner $T$, et al. Diagnosis and management of AML in adults: 2017 ELN recommendations from an international expert panel. Blood. 2017;129:424-47.

13. Bacigalupo A, Ballen K, Rizzo D, Giralt S, Lazarus H, Ho V. et al. Defining the intensity of conditioning regimens: working definitions. Biol Blood Marrow Transplant. 2009;15:1628-33.

14. Glucksberg $H$, Storb R, Fefer A, Buckner CD, Neiman PE, Clift RA, et al. Clinical manifestations of graft-versus-host disease in human recipients of marrow from HL-A-matched sibling donors. Transplantation. 1974;18:295-304.

15. Filipovich AH, Weisdorf D, Pavletic S, Socie G, Wingard JR, Lee SJ, et al. National Institutes of Health consensus development project on criteria for clinical trials in chronic graft-versus-host disease: I. Diagnosis and staging working group report. Biol Blood Marrow Transplant. 2005;11:945-56.

16. Sorror ML, Sandmaier BM, Storer BE, Maris MB, Baron F, Maloney DG, et al. Comorbidity and disease status-based risk stratification of outcomes among patients with acute myeloid leukemia or myelodysplasia receiving allogeneic hematopoietic cell transplantation. J Clin. Oncol. 2007;25:4246-54.

17. Lindsley RC, Mar BG, Mazzola E, Grauman PV, Shareef S, Allen SL, et al. Acute myeloid leukemia ontogeny is defined by distinct somatic mutations. Blood. 2015;125:1367-76.

18. Lin TL, Rizzieri DA, Ryan DH, Schiller GJ, Kolitz JE, Uy GL, et al. Older adults with newly diagnosed high-risk/secondary AML who achieved remission with CPX351: phase 3 post hoc analyses. Blood Adv. 2021;5:1719-28.

19. Paiva B, Vidriales MB, Sempere A, Tarín F, Colado E, Benavente C. et al. PETHEMA (Programa para el Estudio de la Terapéutica en Hemopatías Malignas) Cooperative Study Group. Impact of measurable residual disease by decentralized flow cytometry: a PETHEMA real-world study in 1076 patients with acute myeloid leukemia. Leukemia. 2021;35:2358-70. https://doi.org/10.1038/s41375-02101126-3.

20. DiNardo CD, Jonas BA, Pullarkat V, Thirman MJ, Garcia JS, Wei AH, et al. Azacitidine and Venetoclax in previously untreated acute myeloid leukemia. N. Engl J Med. 2020;383:617-29.

21. Cortes JE, Heidel FH, Hellmann A, Fiedler W, Smith BD, Robak T, et al. Randomized comparison of low dose cytarabine with or without glasdegib in patients with newly diagnosed acute myeloid leukemia or high-risk myelodysplastic syndrome. Leukemia. 2019;33:379-89.

22. Lindsley RC, Gibson CJ, Murdock HM, Stone RM, Cortes JE, Uy GL, et al. Genetic characteristics and outcomes by mutation status in a Phase 3 study of CPX-351 versus $7+3$ in older adults with newly diagnosed, high-risk/secondary acute myeloid leukemia (AML). Blood. 2019;134:15-15.

23. Stölzel F, Platzbecker U, Mohr B, Röllig C, Middeke JM, Thiede C, et al. Early intervention with allogeneic hematopoietic cell transplantation during chemotherapy-induced aplasia in patients with high-risk acute myeloid leukemia. Leukemia. 2013;27:2068-72.

\section{ACKNOWLEDGEMENTS}

This work was supported by a restricted grant of JAZZ Pharmaceuticals.

\section{AUTHOR CONTRIBUTIONS}

Study conception and design and collection and assembly of data: CR, FS, TSc, and $J M M$. Provision of patient data: all authors. Data analysis and interpretation: $C R, F S$, UG, ML, LH, TSa, and JMM. Manuscript writing: CR, FS, HD, KG, TSC, and JMM. Final approval of the manuscript: all authors.

\section{FUNDING}

Open Access funding enabled and organized by Projekt DEAL.

\section{COMPETING INTERESTS}

T Schroeder: advisory boards, lecture fees from JAZZ Pharmaceuticals Germany. JMM: personal fee, advisory board from JAZZ Pharmaceuticals Germany. HD: Advisory Board with honoraria: AbbVie, Agios, Amgen, Astellas, Astex Pharmaceuticals, AstraZeneca, Berlin-Chemie, BMS, Celgene, GEMoaB, Gilead, Helsinn, Janssen, Jazz, Novartis, Oxford Biomedica, Roche; clinical research funding: Agios, Amgen, Astellas, Bristol Myers Squibb, Celgene, Jazz Pharmaceuticals, Novartis, Pfizer. KG: Advisory Board with honoraria: AbbVie, JAZZ, BMS/Celgene Deutschland $\mathrm{GmbH}$, Alexion; research funding: BMS/Celgene. T Sauer: Advisory Board with honoraria: AbbVie, Takeda, Astellas. CR: financial travel support: JAZZ Pharmeceuticals and BMS/Celgene Deutschland $\mathrm{GmbH}$; lecture fees: BMS/Celgene Deutschland $\mathrm{GmbH}$. GK: received lecture fees from BMS/Celgene Deutschland GmbH, Novartis, Jazz Pharmaceuticals and Janssen-Cilag GmbH. FS: advisory board with honoraria: JAZZ Pharmaceuticals, AbbVie, medac $\mathrm{GmbH}$. Ulrich Germing: research funding: BMS/Celgene Deutschland $\mathrm{GmbH}$, Novartis.

\section{ADDITIONAL INFORMATION}

Supplementary information The online version contains supplementary material available at https://doi.org/10.1038/s41408-021-00558-5.

Correspondence and requests for materials should be addressed to Thomas Schroeder or Jan Moritz Middeke.

Reprints and permission information is available at http://www.nature.com/ reprints

Publisher's note Springer Nature remains neutral with regard to jurisdictional claims in published maps and institutional affiliations.

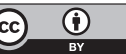

Open Access This article is licensed under a Creative Commons Attribution 4.0 International License, which permits use, sharing, adaptation, distribution and reproduction in any medium or format, as long as you give appropriate credit to the original author(s) and the source, provide a link to the Creative Commons license, and indicate if changes were made. The images or other third party material in this article are included in the article's Creative Commons license, unless indicated otherwise in a credit line to the material. If material is not included in the article's Creative Commons license and your intended use is not permitted by statutory regulation or exceeds the permitted use, you will need to obtain permission directly from the copyright holder. To view a copy of this license, visit http://creativecommons. org/licenses/by/4.0/.

(c) The Author(s) 2021 